Trabajos y Comunicaciones, 2da. Época, Nº 45, e035, marzo 2017. ISSN 2346-8971

Universidad Nacional de La Plata.

Facultad de Humanidades y Ciencias de la Educación.

Departamento de Historia

\title{
Archivo del Poder Legislativo de Mendoza. Los Diarios de sesiones legislativas como fuente documental
}

\author{
Archive of the legislative branch of Mendoza. Daily legislative \\ sessions as a documentary source
}

\author{
Natalia Luis * \\ * CONICET - Instituto de Ciencias Humanas, Sociales y Ambientales - \\ Centro Científico Tecnológico - Mendoza, Argentina | nluis@mendoza-conicet.gob.ar
}

PALABRAS CLAVE

Archivo

Fuente legislativa

Diarios de sesiones

Mendoza

KEYWORDS

Archive

Legislative Source

Daily sessions

Mendoza

\section{RESUMEN}

En este trabajo se describen las características generales del Archivo del Poder Legislativo de Mendoza. Se alude al material disponible, a las posibilidades de acceso y al estado de conservación documental del mismo. Por otro lado, se hace referencia a la particularidad de los diarios de sesiones legislativas como fuente documental. 
Esto es debido al desconocimiento de la existencia de algunas fuentes legislativas en la Provincia de Mendoza, y del mismo modo, por la falta de difusión respecto al funcionamiento del Archivo legislativo. Se considera entonces de importancia poner en valor los escritos legislativos como fuente documental y facilitar al investigador que lo requiera la información necesaria para su búsqueda y consulta.

En este sentido, cabe destacar que las fuentes legislativas de Mendoza se encuentran conservadas en un depósito de carácter privado en calle Rodríguez Peña (Departamento Godoy Cruz), que guarda, entre documentos de otras instituciones, material proveniente de la Cámara de Senadores y de la Cámara de Diputados de la Provincia. En el Banco de Archivos Sociedad Anónima (BASA) no se permite el ingreso al público en general, sólo accede el personal del establecimiento. De toda la documentación producida por las Cámaras legislativas, una pequeña parte es administrada por la Biblioteca de la Legislatura de Mendoza, ubicada en la sede de la Cámara de Diputados en calle Peatonal Sarmiento de la Ciudad de Mendoza. Pero para acceder a la mayoría de las fuentes, hay que apelar al Archivo Legislativo, ubicado en la sede de la Cámara de Senadores en calle Gutiérrez número 38, Mendoza. Ambos edificios se encuentran en el centro administrativo-comercial de la ciudad.

Por ello, si a lo largo de una investigación se desea consultar fuentes legislativas, es imprescindible indagar previamente qué material es administrado en cada Institución. Esto se ve facilitado debido a que ambas poseen un catálogo sobre el material disponible. En definitiva, son dos los repositorios a los que el historiador debe apelar para la consulta de documentos legislativos, a saber, la Biblioteca de la Legislatura, ubicada en el edificio de la Cámara de Diputados, y el Archivo Legislativo, en la sede de la Cámara de Senadores de la provincia de Mendoza.

\section{Biblioteca de la Legislatura de Mendoza}

En esta dependencia se resguardan y administran diversas fuentes, la mayoría son escritos del siglo $\mathrm{XX}, \underline{1}$ representadas tanto por documentos oficiales de gobierno, como por ejemplo el Boletín oficial de Mendoza y el Boletín oficial Nacional, como material de prensa gráfica. De manera específica, el material legislativo que custodia incluye proyectos de ley, tanto nacionales como provinciales, anales de legislación argentina, trámites parlamentarios, Digesto Ediar, Diarios de sesiones legislativas de Diputados y Senadores Nacionales, y algunos Diarios de sesiones legislativas de la Cámara de Diputados y Senadores de la Provincia de Mendoza.

En relación a la documentación legislativa y los escritos de gobierno, se ofrece un catálogo en el cual se detalla a partir de un número de caja, el tipo de documento y periodo histórico del material que la misma contiene. Excepto el material hemerográfico y algunos anales de legislación, los documentos se conservan en un depósito privado, como se mencionó al comienzo. Por ello, el investigador debe pedir con antelación el material al personal administrativo de la Biblioteca para que este sea solicitado y trasladado a la sala de lectura, lugar en el cual puede ser consultado. $\underline{2}$

Una vez que el material se encuentra disponible en la sala de lectura, el interesado debe cumplir algunas normas relacionadas con el correcto manejo de los documentos y la conservación del material, entre estas, se incluye la prohibición de emplear tinta o cualquier otro elemento que afecte 
la integridad del documento, así como la imposibilidad de consumir alimentos y/o bebidas cerca de los escritos. Además, si bien no es obligatorio, el personal administrativo recomienda el uso de guantes de látex.

Ahora bien, esta dependencia gestiona una pequeña proporción de los escritos legislativos existentes en Mendoza, el resto, es decir la mayoría de la documentación legislativa, es administrada por el Archivo de la Cámara de Senadores de la Provincia de Mendoza. Si bien algunos ejemplares de diarios de sesiones y demás documentos legislativos se encuentran catalogados en ambos establecimientos, la mayoría del material no se encuentra repetido y es administrado exclusivamente por una u otra dependencia, por ello es necesario consultar ambas para realizar una búsqueda completa.

\section{Archivo de la Cámara de Senadores de la Provincia de Mendoza}

El Archivo de la Cámara de Senadores de Mendoza no es un archivo histórico ni reúne las condiciones necesarias para resguardar documentos antiguos. Se trata más bien de un repositorio de guarda administrativo que se ocupa tanto de escritos históricos como contemporáneos.

No es una oficina amplia, sino una dependencia dentro del edificio donde se localiza la sede de la Honorable Cámara de Senadores, en la cual se encuentran las oficinas de los senadores provinciales. Tiene problemas de espacio y de recursos por lo cual la mayoría de la documentación es conservada en un depósito Privado, tal como se mencionó al comienzo.

En este sentido, si bien el Archivo es de acceso libre y cualquier persona puede ingresar a consultar, se debe solicitar el material específico a investigar mediante una nota formal dirigida al Jefe del Archivo Legislativo de la Cámara de Senadores, Samuel Martinelli. De ese modo, en primer lugar se debe acceder al catálogo del material archivado. El mismo presenta los escritos ordenados según su tipología y datación temporal. Precisamente, una vez que el material a consultar es elegido y se presenta la nota, el encargado del archivo solicita a su vez el material al depósito privado, el cual es trasladado al Archivo de la Cámara de Senadores para ser consultado.

Ahora bien, cuando el material llega a la oficina, no se le informa ninguna directiva al usuario y no hay personal que controle el correcto uso de los documentos en pos de su preservación. Esto lleva a que se deterioren rápidamente los escritos.

El material archivado incluye proyectos de ley, historial legislativo de la Cámara de Diputados, de Senadores y del Poder Ejecutivo de Mendoza, expedientes de la Cámara de Diputados, recopilación de leyes, libro de actas, resoluciones de la presidencia de la Cámara, resoluciones del cuerpo de Senadores, oficios intercambiados entre Poder Ejecutivo y Poder Legislativo, peticiones de particulares y de diversas asociaciones a la Legislatura en general y a legisladores en particular, diarios de sesiones legislativas de la Cámara de Diputados y de la Cámara de Senadores de la Provincia de Mendoza, y documentación sobre cuestiones administrativas y económicas (presupuesto, libro de gastos, libro caja, libro de impuestos, planillas de sueldo) de la Honorable Legislatura. 


\section{Estado de la documentación}

Con respeto al estado de la documentación legislativa, se observa que la mayoría del material se encuentra deficientemente conservado. Los escritos están almacenados en un depósito privado en cajas de cartón expuestas al polvo y a otros agentes corrosivos. En algunos documentos se ha detectado hongos y humedad, lo cual dificulta su consulta. Además, si bien el material está clasificado según su tipología (Proyectos de ley, diarios de sesiones, actas legislativas, expedientes, presupuesto legislativo, y otros), en algunas cajas no se encuentra el material que debería estar ya sea por robo o desorden administrativo. Por otro lado, se ha detectado pérdida de cajas completas con todo el material.

Asimismo, no se han implementado políticas de reparación y/o restauración de los escritos dañados. Además, la documentación no está digitalizada y el catálogo del material se presenta en formato papel, sin dar la posibilidad de búsqueda mediante computadora para los investigadores que deseen realizarlo.

\section{Diarios de sesiones legislativas como fuente documental}

De toda la documentación legislativa existente en Mendoza, interesa de manera particular los diarios de sesiones legislativas. Los mismos dan cuanta de las discusiones parlamentarias en torno a los diferentes proyectos de ley, y en este sentido, a partir de ellos se puede analizar no sólo las medidas implementadas, sino las diferentes propuestas legislativas que no llegaron a efectivizarse pero que también forman parte del proyecto político de los diferentes gobiernos. Del mismo modo, los diarios de sesiones clarifican el entendimiento de las diferencias partidarias e intra-partidarias a partir del análisis de la argumentación esgrimida por los legisladores en torno a los proyectos de ley.

Ahora bien, la consulta de los diarios de sesiones legislativas se dificulta en Mendoza por dos causas. En primer lugar, debido a que no están conservados todos los diarios de sesiones, $\underline{3}$ ello, sumado a la pérdida o extravío de algunos de ellos, obstaculiza el estudio de la continuidad en el tratamiento de los diferentes proyectos de ley por los legisladores; en segundo lugar, del material a consultar, en algunas ocasiones sólo se encuentra conservada la versión manuscrita (no taquigrafiada), con la consecuente dificultad en el entendimiento del escrito y en la cantidad de tiempo invertido, ya que se trata de documentos muy extensos.

Sin embargo, se considera que los diarios de sesiones, así como los demás documentos legislativos, constituyen una fuente original a explorar, que ha sido escasamente consultada y que da cuenta de la realidad política, económica, cultural y social de la historia de Mendoza no visible a partir de otras fuentes documentales.

\section{Notas}

1 La Biblioteca de la Legislatura también conserva material del siglo XIX, tales como diarios de sesiones legislativas, proyectos de ley y algunos ejemplares del Boletín Oficial, aunque la proporción es mucho menor en relación a la documentación resguardada del siglo XX.

$\underline{2}$ El pedido se realiza oralmente, de manera informal, a diferencia del material solicitado en el Archivo de la Cámara de Senadores. 
$\underline{3}$ Si bien esto depende del periodo histórico a investigar se ha detectado que no se conservan muchos diarios de sesiones del siglo XX. 